\title{
and still going strong
}

In the evening of 24 November 1959, a jubilant crowd in the control room of the sparkling new CERN Proton Synchrotron witnessed a proton beam accelerated to $24 \mathrm{GeV}$, at the time a new world record. The pride of Europe, the machine was the first to use the then new principle of alternating gradient focusing, and came into operation a full year ahead of its US counterpart, the Brookhaven Alternating Gradient Synchrotron.

The availability of the world's most powerful accelerator was a good start for high energy physics at the world's first international Laboratory. The PS has long since ceased to hold the world particle beam energy record, but thanks to the vision of its designers and a series of ingenious improvements which have provided increased performance and reliability, it remains the kingpin of CERN's installations, the largest and most versatile complex of high energy par- ticle accelerators in the world.

After exploiting the original configuration to the full, the second decade of the machine's operation saw the arrival of the $800 \mathrm{MeV}$ Booster synchrotron which increased the injection energy and hence the available beam intensity, and a new radiofrequency accelerating system. Later improvements included a new $50 \mathrm{MeV}$ linac alongside the old one, new pole face windings for the magnets, the replacement of the original mercury arc rectifiers by solid-state ones, a new modular control system to take care of the complicated new modes of operation of the machine, and a radiofrequency quadrupole to replace the classic Cockcroft-Walton electrostatic pre-injector at the 'old' linac.

However the biggest changè came in the role of the machine itself. Originally, the PS provided particle beams directly to the adjacent exper-

\section{Beam exercises}

The evolution of the CERN $P S$. In blue, the proton circuit, including the two linac injectors (top) and the adjoining Booster. The principal extracted beams fan out to the right, serving, from the top, the low energy neutrino beam, the Intersecting Storage Rings (ISR - now closed) and the $450 \mathrm{GeV}$ Super Proton Synchrotron (below, not visible on plan), and the antiproton target.

In red, the antiproton circuit. The secondary antiprotons are stored in the Antiproton Accumulator (AA) and loop back to the PS. At the bottom, the extracted beam branches in two to serve the ISR and the SPS. At the top, the low energy antiprotons emerge for the LEAR ring.

In green, the future. Top left, electrons and positrons arrive from the new $L E P$ injector. Before reaching $\angle E P$, the electrons pass to the SPS along the same path as the antiprotons, while positrons follow the proton path, along which the PS will also feed oxygen ions to the SPS.

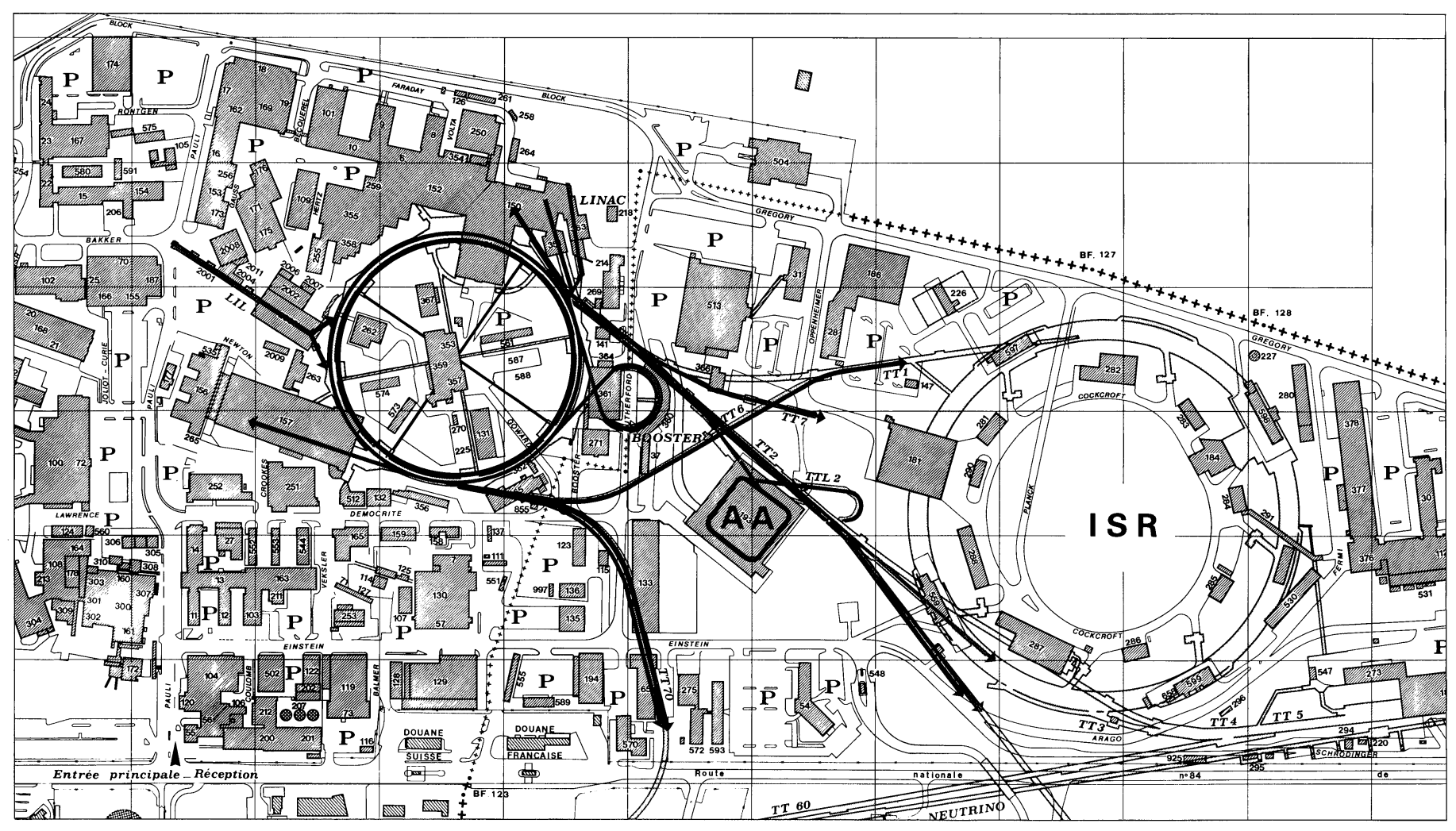



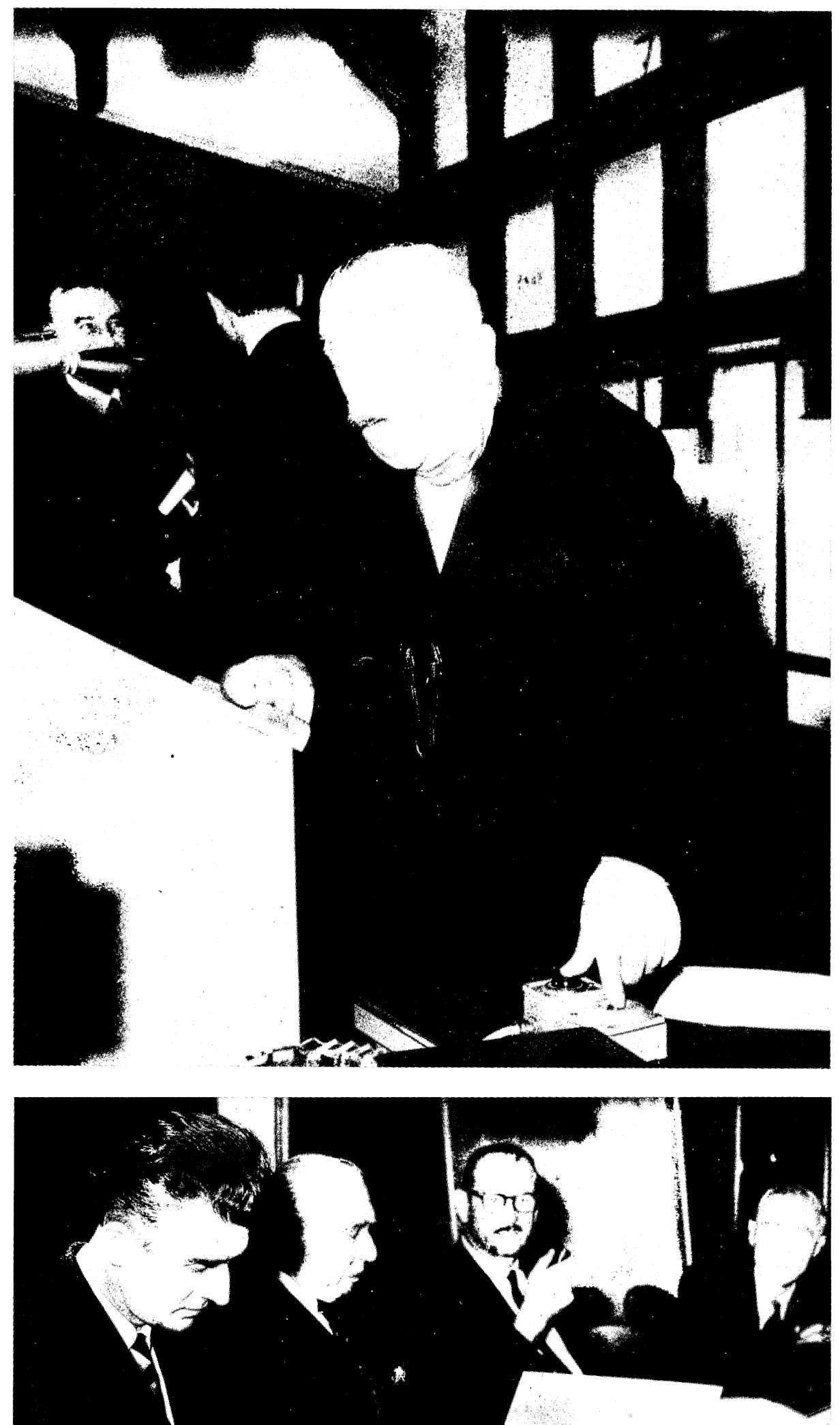

imental areas. But over the years it was increasingly called on to supply particles to other machines, first the Intersecting Storage Rings in 1971 and then in 1976 the SPS $400 \mathrm{GeV}$ Super Proton Synchrotron.

With the advent of the CERN antiproton project, the PS became in 1981 the world's first antiproton synchrotron. Working in this mode, the PS takes $3.5 \mathrm{GeV}$ antiprotons from the Antiproton Accumulator (AA), accelerating them to $26 \mathrm{GeV}$ ready for injection into the SPS.

As if this were not enough, another antiproton requirement emerged. For the LEAR Low Energy Antiproton Ring, the PS has to take on yet another very different role, that of a particle decelerator rather than an accelerator. The precious antiprotons from the AA are slowed down from $3.5 \mathrm{GeV}$ to a kinetic energy near $200 \mathrm{MeV}$

In addition, there were alpha particles for the ISR, new beams for low energy neutrino experiments, new test beams... The valiant PS never failed to rise to the occasion.

The machine's big moment for physics came in 1973 with the discovery of the neutral current in neutrino interactions using the Gargamelle bubble chamber. With the arrival on the scene of the SPS, many of the big detectors migrated to the bigger machine. The PS retained a faithful band of experimenters, and in the last few years the novel LEAR programme has attracted a flock of several hundred physicists to the PS many of them new to CERN.

The PS provides per pulse over a thousand times the intensity delivered back in 1959 and works in complicated 'supercycles' to cater for its

Another shot from the inauguration showing left to right, John Adams, Niels Bohr, Ed McMillan, and J. Robert Oppenheimer.

(Photo CERN 2065) 


\section{Evolution of CERN Proton Synchrotron parameters}

1959

Intensity

Pulse rate

Energy

Injector

Accelerated particles

Magnet Power Supply
Voltage
Current
Cycle type

\section{Accelerating systems}

Accelerating cavities

RF power

\section{Vacuum System \\ Pumps}

Average pressure

Volume under vacuum

\section{Injection system}

$50 \mathrm{MeV}$

$800 \mathrm{MeV}$ protons

$3.5 \mathrm{GeV}$ antiprotons

$600 \mathrm{MeV}$ electrons*

Beam utilization systems design $10^{10} \mathrm{p} / \mathrm{p}$

achieved $3 \times 10^{10} \mathrm{p} / \mathrm{p}$

1 per 3 to $5 \mathrm{~s}$

design $25 \mathrm{GeV}$

achieved $28 \mathrm{GeV}$

$50 \mathrm{MeV}$ Linac

Protons

Motor generator set with one mercury arc rectifier

$5400 \mathrm{~V}$

$6000 \mathrm{~A}$

single cycle with 20 ms peak

16 units of $10 \mathrm{kV}$ each tunable from 2.5 to $9.5 \mathrm{MHz}$

$16 \times 6 \mathrm{~kW}$

60 oil diffusion

4. $10^{-6}$ torr

$9 \mathrm{~m}^{3}$

single turn with 1 electrostatic inflector and 3 pulsed inflectors

2 internal targets for South Hall (target 1) and North Hall (target 6)

1 main user plus parasite tests, emulsion exposures and irradiation chemistry

Exploratory counter experiments, $32 \mathrm{~cm}$ hydrogen bubble chamber
1984 ( ${ }^{*}$ under development)

$2.2 \times 10^{13} \mathrm{p} / \mathrm{p}$

1 per 1.2 to $2.4 \mathrm{~s}$

0.2 to $25 \mathrm{GeV}$ (kinetic)

Injector 1 - $800 \mathrm{MeV}$ four-ring Booster (PSB) fed by new $50 \mathrm{MeV}$ Linac Injector 2 - Antiproton Accumulator Injector $3^{*}$ - Electron Positron Accumulator

Protons, deuterons, alphas, antiprotons, electrons*, positrons *, oxygen*

Motor generator set with two thyristor rectifiers

$9000 \mathrm{~V}$

$6000 \mathrm{~A}$

Supercycle adapted to the user: $14 \mathrm{GeV}$ for SPS, 26 for SPS Collider and AA, 24 for experimental physics, and decelerating cycles for LEAR antiprotons

10 units of $20 \mathrm{kV}$ each tunable from 2.5 to $9.5 \mathrm{MHz}$

8 units of $50 \mathrm{kV}$ each tuned at $200 \mathrm{MHz}$, 2 units of $500 \mathrm{kV}$ each at $114 \mathrm{MHz}$

$$
\begin{aligned}
& 10 \times 100 \mathrm{~kW} \text { (at } 9.5 \mathrm{MHz}) \\
& 8 \times 20 \mathrm{~kW} \text { (at } 200 \mathrm{MHz} \text { ) } \\
& 2 \times 160 \mathrm{~kW} \text { (at } 114 \mathrm{MHz} \text { ) }
\end{aligned}
$$

139 ion and 14 turbomolecular (New vacuum chamber ${ }^{*}$ )

2. $10^{-8}$ torr

$16 \mathrm{~m}^{3}$

single turn with septas, fast deflectors and orbit deformation

Slow Extraction for East Hall Fast extraction for SPS/AA and with decelerated particles for LEAR Continuous extraction for SPS

Test beams in East Area. 


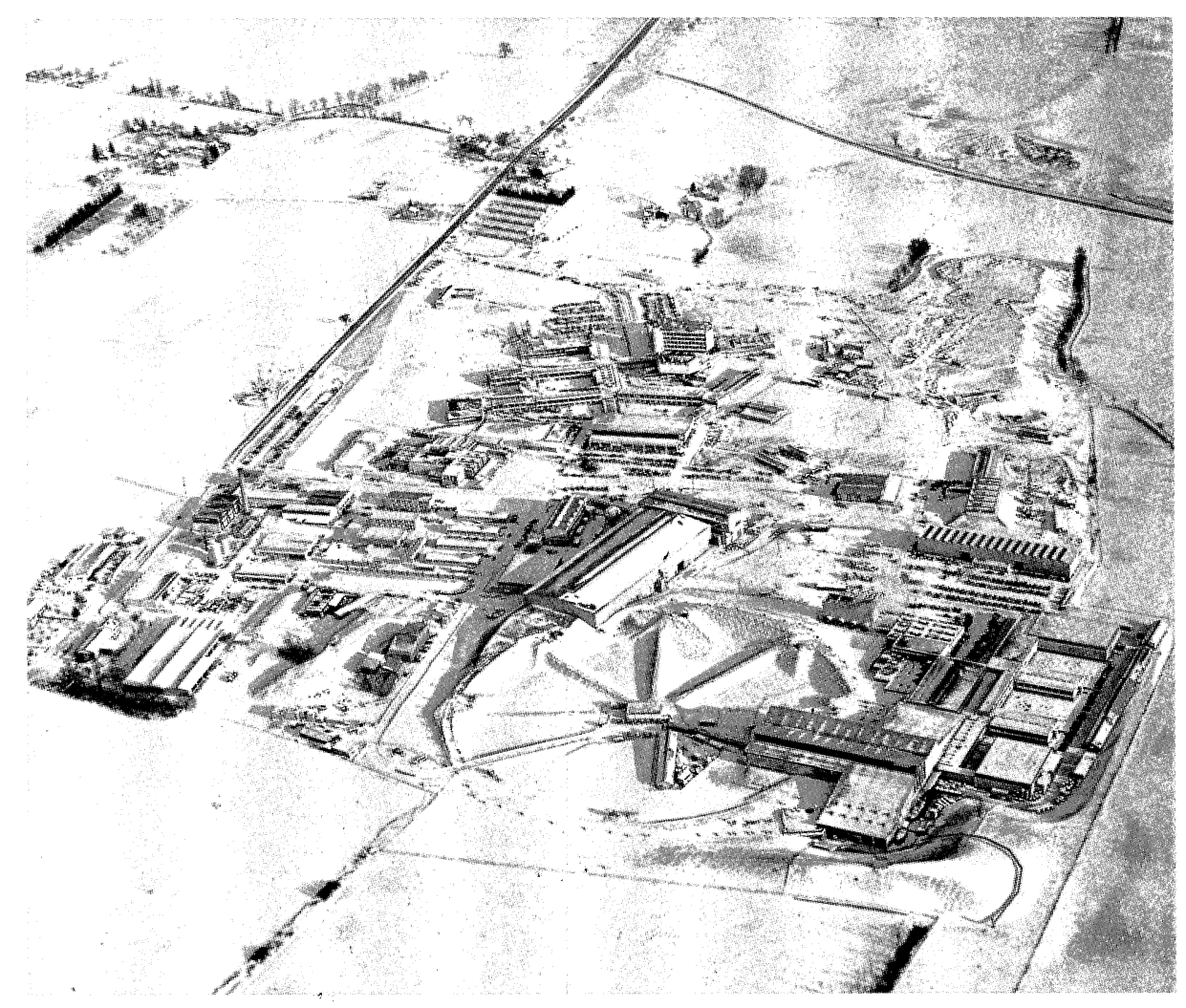

An early aerial view (1962) of the CERN site showing the PS ring with its experimental halls to the south (right) and to the east.

(Photo CERN 256.1.63)

ity of this faithful CERN workhorse bear tribute to the sound engineering work of the team which designed and built it in those far-off days, and to the imagination and resourcefulness of those who have developed it into the unique facility it is today.

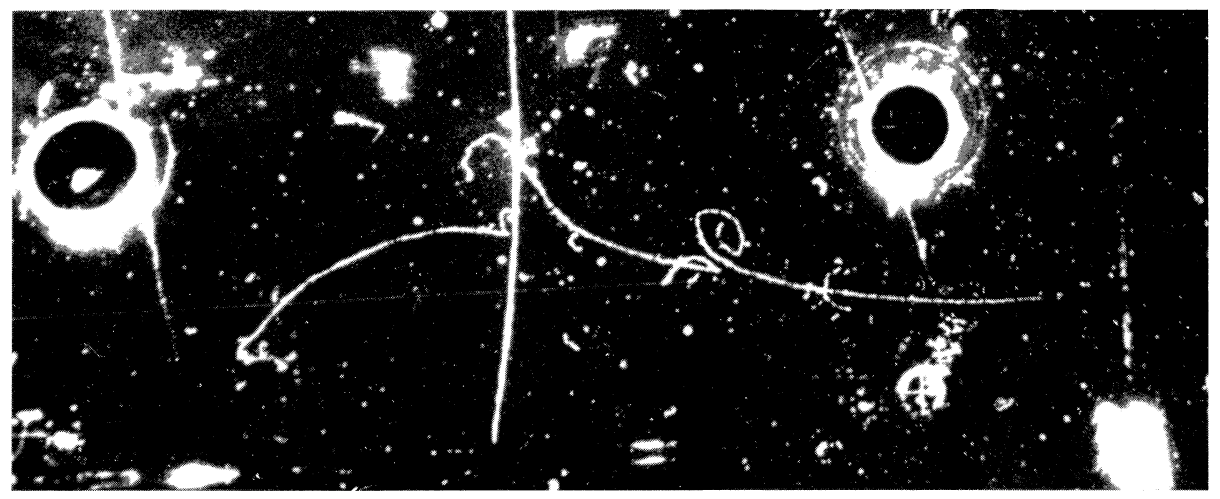

Left, in 1973 came the big physics discovery at the PS - the neutral current of the weak interaction, seen in neutrino interactions in the Gargamelle heavy liquid bubble chamber.

wide variety of users. While one customer, the ISR, has disappeared, for the future the PS will have to handle beams of 'heavy' ions (oxygen 16) for a new generation of experiments, and the electron and positron bunches for the LEP collider, now under construction. For this, the PS will receive particles from the new EPA electron-positron accumulator taking shape in the south-east sector of the machine.

No proposal for a successor to the PS has yet appeared on the horizon, so that the machine, now at the hub of a complex particle factory of ten interconnected accelerators, seems likely to be still providing CERN's particles when the 21 st century arrives. The flexibility and astounding reliabil-

\footnotetext{
The Antiproton Accumulator, the heart of the CERN antiproton scheme, which provides the PS with antiprotons at $3.5 \mathrm{GeV}$.
}

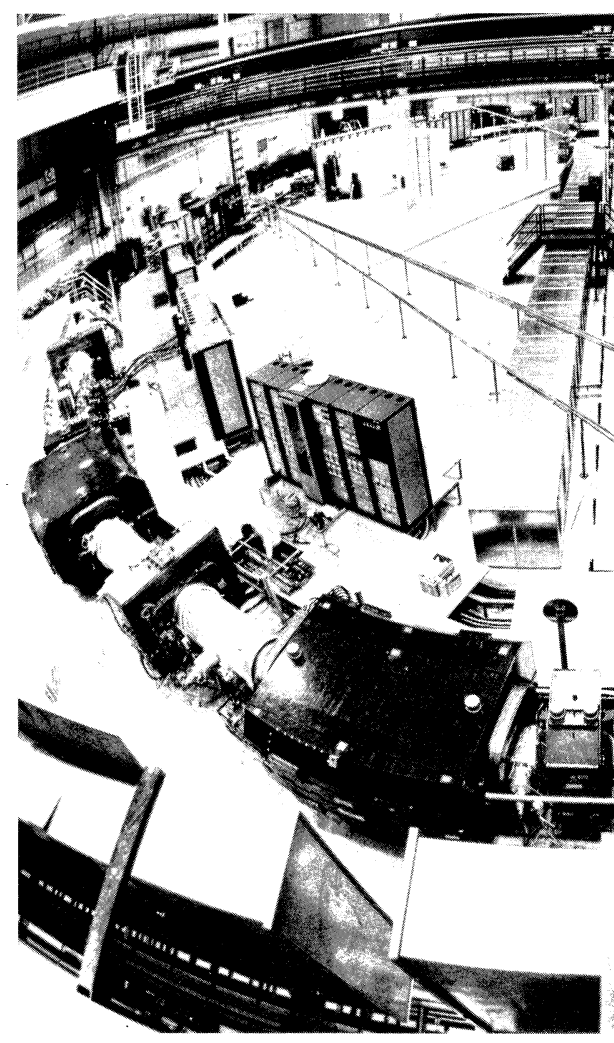

(Photo CERN 582.10.80) 